\title{
Electron-Tomography of a Temperature-Sensitive Morphogenic Mutant of Rous Sarcoma Virus (RSV)
}

\author{
C. Butan*\#, P.M. Lokhandwala**, D.C. Winkler*, R.C. Craven** and A.C. Steven* \\ * Laboratory of Structural Biology, National Institute for Arthritis, Musculoskeletal \\ and Skin Diseases, National Institutes of Health, Bethesda, Maryland 20892 \\ ** Department of Microbiology and Immunology, The Pennsylvania State University \\ College of Medicine, Hershey, Pennsylvania 17033 \\ \# Present address: Laboratory of Cell Biology, National Cancer Institute, National \\ Institutes of Health, Bethesda, Maryland 20892
}

During retrovirus maturation, the Gag precursor polyprotein is processed into its matrix (MA), capsid (CA), and nucleocapsid (NC) components. The CA subunits assemble into the capsid shell containing the RNA genome and replication enzymes. Its structure is thought to be critical for viral infectivity. CA has two domains whose amino acid sequences are conserved only in a 20-residue stretch in the $\mathrm{C}$-terminal domain called the major homology region (MHR). It has been observed that mutations in the MHR impair virus infectivity $[1,2]$. To investigate this phenomenon, we have used electron cryotomography to characterize the structure of temperature-sensitive RSV virions bearing a primary point mutation in the MHR (F167Y) that essentially eliminates infectivity together with a secondary compensating mutation, A38V.

Tomography was performed as described [3]. Drops of isolated virions mixed with 10$\mathrm{nm}$ gold particles were applied to holey carbon films, thinned by blotting, vitrified, and transferred into a cryo-holder. Tilt series were acquired on a Tecnai-12 microscope, equipped with an energy filter that was operated in the zero-energy-loss mode with a slit width of $20 \mathrm{eV}$. Images were recorded on a $2048 \times 2048$-pixel CCD camera (Gatan) at $38,000 \times$ magnification $(0.78 \mathrm{~nm}$ pixels). The angular range covered was typically from $60^{\circ}$ to $+60^{\circ}$ in increments of $1^{\circ}$. Data were recorded using SerialEM [4]. The nominal defocus was $-6 \mu \mathrm{m}$, and the total dose delivered to the specimen was $\sim 60 \mathrm{e}^{-} / \AA^{2} .10$ tomographic reconstructions containing a total of about 200 particles were calculated, using IMOD [5].

When the double mutant is grown at the non-permissive temperature of $42^{\circ} \mathrm{C}$, the virions produced are more variable in size than the wild-type and have fewer glycoprotein spikes. Although Gag is processed normally, capsid formation during maturation is inhibited (Fig. 1). The infectious virions formed at the permissive temperature $\left(37^{\circ} \mathrm{C}\right)$, however, are similar to wild-type in both size and glycoprotein content, and capsid assembly is restored, albeit with an altered range of polymorphism. The mutant produces fewer tubular capsids and more closed filled irregular polyhedral capsids than wild-type (Fig. 2). Also, these polyhedral capsids tend to be larger than wild-type capsids (Fig. 3). These observations suggest that at the non-permissive temperature, the conformation of the mutant CA does not support ordered assembly but at the permissive temperature, this protein is even more morphogenically competent than wild-type CA, to judge by the higher incidence of closed, filled, polyhedral capsids. 


\section{References}

[1] P.M. Lokhandwala et al., Virology, 376 (2008) 191

[2] J.G. Purdy et al.,, J. Virol. 82 (2008) 5951.

[3] C. Butan et al.,, J. Mol. Biol.. 376 (2008) 1168.

[4] D.N. Mastronarde, J. Struct. Biol., 152 (2005) 36.

[5] J.R. Kremer, J. Struct. Biol., 116 (1996) 71.

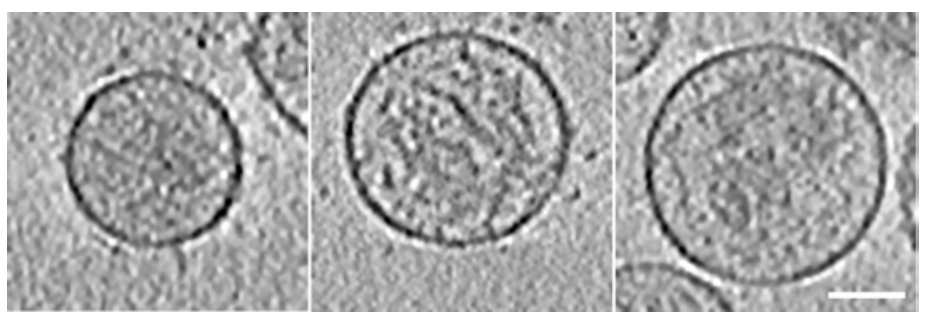

Fig 1. Electron cryo-tomography of FY/AV virions grown at the non-permissive temperature of $42^{\circ} \mathrm{C}$. Gallery of central sections through three representative virions. Scale bar, $50 \mathrm{~nm}$.

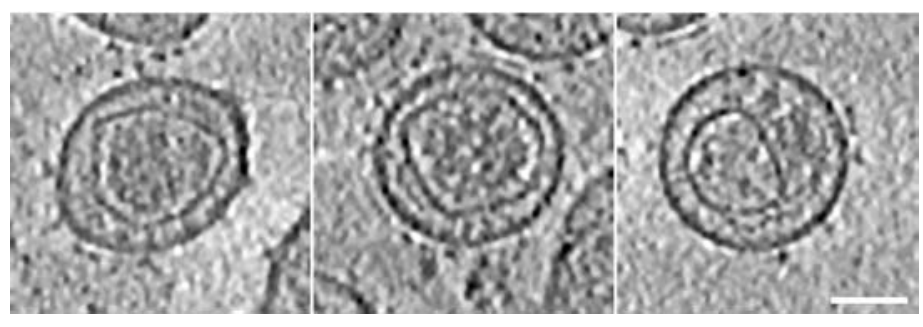

Fig 2. Electron cryo-tomography of FY/AV virions grown at the permissive temperature of $37^{\circ} \mathrm{C}$. Gallery of central sections through three representative virions. Scale bar, 50 $\mathrm{nm}$.

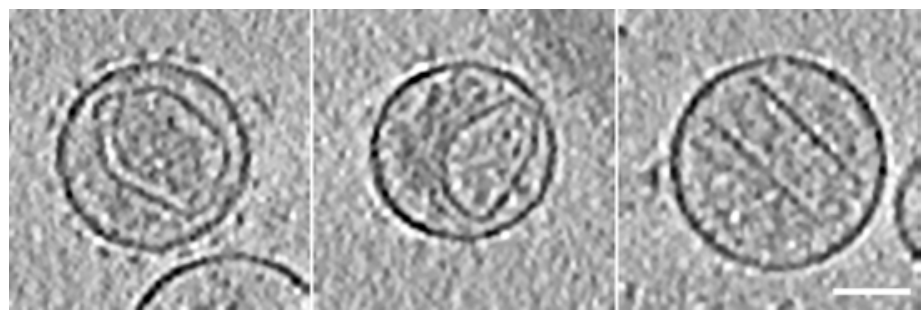

Fig 3. Electron cryo-tomography of wild-type virions. Gallery of central sections through three representative virions [3]. Scale bar, $50 \mathrm{~nm}$. 